\title{
Enhanced Iontophoretic Delivery of Magnesium Ascorbyl 2-Phosphate and Sodium Fluorescein to Hairless and Hairy Mouse Skin
}

\author{
Min Young Kang, Su Hee Kim, Young Kwan Sung, Moonkyu Kim, Jung Chul Kim, Insook Han* \\ Department of Immunology, School of Medicine, Kyungpook National University, Daegu, South Korea. \\ Email: *ishan60@hanmail.net
}

Received July $16^{\text {th }}, 2012$; revised August $18^{\text {th }}, 2012$; accepted August $29^{\text {th }}, 2012$

\begin{abstract}
We recently reported that L-ascorbic acid 2-phosphate (AP) stimulates the growth of human dermal papilla (DP) cells, induces secretion of IGF-1 from the DP cells to promote hair shafts elongation in cultured human hair follicles, and triggers early progression from the telogen to anagen phase in mice. Since the magnesium salt of AP (APMg) is a highly hydrophilic ionic molecule, it is not easy to deliver this reagent to the skin or hair follicles by topical application alone. In order to enhance skin penetration of APMg without changing any molecular properties, a non-invasive iontophoretic delivery method was introduced. Iontophoresis of the negatively charged APMg under the electrode bearing same charge (cathode) significantly enhanced the in vitro penetration of APMg into a Franz cell equipped with mouse dorsal skin. In contrast, iontophoretic movement with the anode inhibited APMg penetration achieved with passive diffusion alone. The effect of iontophoresis on enhancing the penetration of APMg was also found to be much higher in the skin of hairy mice (3 - 8 times) compared to hairless mice (1.5 - 2.5 times). These findings indicated that iontophoretic movement induced the transfollicular pathway more strongly and effectively than the transdermal pathway. This phenomena was also demonstrated by the in vivo iontophoretic delivery of sodium fluorescein using hairy and hairless mice. The degree of iontophoretic enhancement during APMg penetration was also dependent on various conditions such as current density and application duration.
\end{abstract}

Keywords: L-Ascorbic Acid 2-Phosphate; Sodium Fluorescein; Iontophoresis; Transfollicular Pathway

\section{Introduction}

The mammalian hair follicle is a finely organized, multilayered, and dynamic organ. Hair follicles contain epithelial and dermal components. They undergo a lifelong cyclic transformation via close epithelial-dermal interactions from a resting (telogen) to a growth phase (anagen) with proliferation of hair epithelial cells followed by a regression phase (catagen) leading to hair follicle involution [1]. The size and depth of the follicle is dependent on the hair cycle stage.

L-ascorbic acid (vitamin C) modulates collagen synthesis and acts as an effective antioxidant. This compound is unstable in aqueous solutions; the half-life of ascorbate in culture medium is $1-2 \mathrm{~h}$. It is also susceptible to UV-mediated destruction. These properties limit the universal use of L-ascorbic acid. Thus, a stable derivative, the sodium and magnesium salt of L-ascorbic acid-2-phosphate (APMg), was introduced. APMg is

*Corresponding author. known to stimulate the growth of human dermal fibroblasts and osteoblasts [2]. It also promotes hair growth in in vitro and in vivo experiments [3-5]. The effect of APMg on hair growth is achieved by the high production of IGF-1 along with versican (the target gene of Wnt/ $\beta$-catenin), alkaline phosphatase expression in cultured dermal papilla cells, and early progression of the telogenanagen transition observed in animal experiments. Regardless of its stability and efficacy, APMg has limited topical use since it is also quite water-soluble. Lipidrich stratum corneum is a highly resistant barrier that prevents APMg penetration into the hair follicle and skin.

Two types of AP salts, sodium and magnesium, have been used to explore ways to improve skin penetration using several enhancing methods [6,7]. Iontophoresis has been investigated as a way to enhance skin penetration since an AP salt molecule includes 2 - 3 anions $[6,8]$. Iontophoresis is an effective and non-invasive method which uses mild electric currents that do not damage the skin barrier. Iontophoresis provides an electrical driving force for transport of drugs across the stratum corneum to 
increase skin penetration of drugs. The amounts of drug penetrated into skin depends on the current intensity, duration and the physicochemical properties of the drug molecule. To enhance the iontophoretic movement of drugs further, liposomes were also combined as carriers $[9,10]$.

In the present study, we determine whether iontophoresis is an effective technique for facilitating the transport of magnesium AP salt across the hair follicle. The transfollicular pathway is known to be substantially less resistant to iontophoresis $[11,12]$. In order to demonstrate this, we compared the difference in APMg skin penetration via iontophoresis between hairy and hairless mice. All APMg that successfully penetrated the skin was analyzed by HPLC. Furthermore, the iontophoretic pathway of sodium fluorescein $(\mathrm{NaF})$ was also evaluated in both hairy and hairless mice since the ionic structure of $\mathrm{NaF}$ is similar to that of APMg. A single molecule of $\mathrm{NaF}$ has two oxynions while an APMg molecule has three.

\section{Materials and Methods}

\subsection{Drugs}

L-ascorbic acid phosphate magnesium salt n-hydrate (APMg) and sodium fluorescein $(\mathrm{NaF})$ were obtained from Wako Ltd. (Osaka, Japan) and Sigma (St. Louis, MO, USA), respectively. The conductivity of APMg was measured by Mettler Toledo GmbH (Schwerzenbach, Switzerland).

\subsection{In Vitro Penetration of APMg}

After sacrificing 6 - 7-wk-old female hairless SKH1 mice and 7-wk-old female hairy C57BL/6 mice (Orient Bio, Daejeon, South Korea), dorsal skin with full-thickness was excised and subsequently mounted in a Franz diffusion cell (PermeGear Inc., Hellertown, PA, USA) kept at $37^{\circ} \mathrm{C}$. The donor cell contained $0.5 \mathrm{~mL}$ of an aqueous APMg solution and the acceptor cell included $6 \mathrm{~mL}$ of an isotonic phosphate buffered solution (PBS 1X). APMg that penetrated the skin was collected at regular intervals over 1 - $30 \mathrm{~h}$. The amount of APMg in the samples was determined by HPLC and expressed as a percentage (\%) relative to the applied dosage. For iontophoresis, ionzyme (Environ Cosmeceutics International, Osaka, Japan) was used at $0.2 \sim 0.8 \mathrm{~mA} / \mathrm{cm}^{2}$ after one charge connected to the donor cell and the other to the acceptor cell. The direction of ionic charge was controlled by the polarity.

\subsection{HPLC Analysis of APMg}

The amount of APMg that penetrated and was retained in the skin was analyzed with a Waters HPLC Delta 600 Analytical system (Waters, Milford, MA, USA) with a
UV-VIS detector. The separation column included a C18 bond phase $(5 \mu \mathrm{m}, 4.6 \times 150 \mathrm{~mm}$, SunFire, MA, USA). The mobile phase contained a mixture of $0.02 \mathrm{M}$ phosphate buffer and methanol $(85: 15 \mathrm{v} / \mathrm{v})$ at $\mathrm{pH}$ 3.0. The flow rate was $0.8 \mathrm{~mL} / \mathrm{min}$ and $\mathrm{APMg}$ was detected at 240 and $258 \mathrm{~nm}$. The retention time of APMg was found to be $2.3-2.7 \mathrm{~min}$. The concentration of APMg was calculated according to a normalized graph using standard samples $(100-500 \mu \mathrm{M})$ of APMg.

\subsection{In Vivo Penetration of Sodium Fluorescein}

After euthanizing the 7-week-old female hairless SKH1 and the 5- or 7-week-old female hairy C57BL/6 mice with forane, the dorsal hairs were cut with an electric shaver. Topical application was performed by attaching the gauze containing a $0.1 \%$ aqueous sodium fluorescein $(\mathrm{NaF})$ solution to the dorsal skin of mice. For iontophoresis, the cathode was connected to the gauze and the anode was attached to the belly of the mice. After iontophoresis and post-incubation, the skins were washed with PBS, harvested, and cut into $10-\mu \mathrm{m}$ frozen section using a Cryostat-Microtome (Leica Microsystems, Nussloch Germany). The green fluorescent $\mathrm{NaF}$ in the slides were observed with a fluorescence microscopy (Olympus BX51, Tokyo, Japan) under blue illumination (excitation, $365 \mathrm{~nm}$; emission, $400 \mathrm{~nm}$ ).

\section{Results and Discussion}

\subsection{In Vitro Iontophoretic Permeation of APMg into Hairless Mouse Skin}

APMg has a molecular weight of 278.4 and three oxyanion per molecule (Figure 1). For effective penetration of APMg using iontophoresis, the conductivity was 445 , 884, and $1204 \mathrm{~s} / \mathrm{cm}$ for $0.04,0.12$, and $0.2 \mathrm{M} \mathrm{APMg}$, respectively. Figure 2(a) shows the in vitro penetration of $0.2 \mathrm{M} \mathrm{APMg}$ into hairless mouse skin. The amount of APMg recovered from the Franz donor cell with 1) passive diffusion; 2) iontophoretic movement $\left(0.4 \mathrm{~mA} / \mathrm{cm}^{2}\right.$, $20 \mathrm{~min}$ ) with cathode; and 3) iontophoresis with anode under the same conditions was compared. Iontophoresis with the cathode against the negative charge of the APMg molecule significantly enhanced the in vitro penetration over time while application with the anode resulted in penetration less than that achieved with passive diffusion. This result demonstrated that eletromigration due to electric repulsion between the cathode and negative APMg charge was more important than electroosmosis for the iontophoretic movement of APMg. Passive diffusion through skin was associated with minimum APMg penetration $(0.04 \%-0.14 \%)$ at 1 and 5 h. In contrast, application of iontophoresis $\left(0.4 \mathrm{~mA} / \mathrm{cm}^{2}\right.$, 


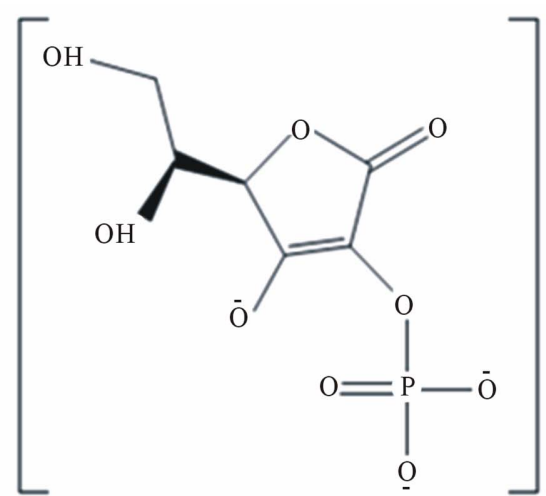

APMg

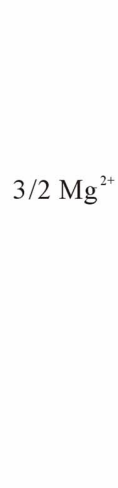

$\mathrm{O}=$<smiles>O=C([O-])c1ccccc1-c1c2ccc(=O)cc-2oc2cc([O-])ccc12</smiles>

$\mathrm{NaF}$

Figure 1. Chemical structures of ascorbic acid 2-phosphate magnesium salt (APMg) and sodium fluorescein (NaF).

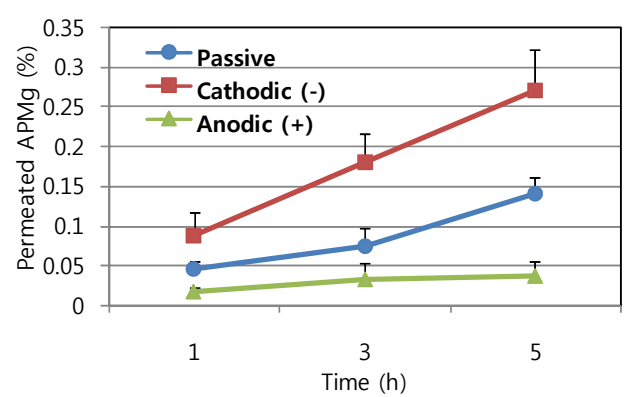

(a)

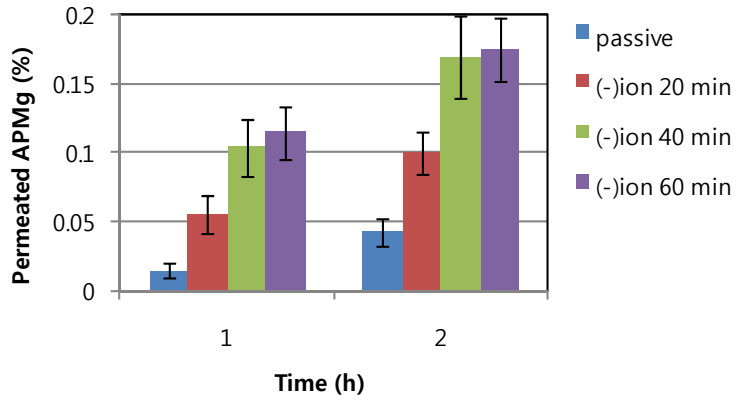

(b)

Figure 2. In vitro penetration of $0.2 \mathrm{M}$ APMg using a Franz cell equipped with hairless skin with or without iontophoresis. A. Permeated APMg with or without iontophoresis at $\pm 0.4 \mathrm{~mA} / \mathrm{cm}^{2}$ for $20 \mathrm{~min}$, B. Effect of iontophoretic duration (20, 40, and 60 min). (a) Passive vs. lomtophoretic penetration; (b) Duration of iontophoresis.

$20 \mathrm{~min}$ ) increased APMg penetration up to $0.09 \%$ $0.27 \%$ (2.0 - 2.5-fold) at the same periods of experiments. Increased penetration was also dependent on the duration of iontophoresis (Figure 2(b)).

\subsection{In Vitro Iontophoretic Permeation of APMg into Hairy Mouse Skin}

Following passive diffusion of APMg through hairy mouse skin of 7-wk-old hairy mice (telogen stage) the level of penetrated APMg was similar to that observed in the hairless skin $(0.02 \%-0.17 \%$ versus $0.04 \%-0.14 \%$ over $1-5 \mathrm{~h}$ ). In contrast, the effect of iontophoresis with cathodic conduct on APMg penetration was excellent in hairy mouse skin; $0.47 \%, 0.75 \%, 0.88 \%$, and $0.86 \%$ of the APMg penetrated at 1, 3,5, and $7 \mathrm{~h}$, respectively, and the resulting enhancement ratio was found to be 23-, 9-, 5.1-, and 3.4-fold over time (Figure 3(a)). The highest enhancement ratio was recorded at $1 \mathrm{~h}$ and the ratio decreased over time. Nevertheless, the enhancement of APMg penetration by iontophoresis was significantly higher in hairy than hairless skin $(3.5-23.0$ - versus 2.0 -
2.5 -fold). These findings indicate that hair follicles play an important role in the iontophoretic enhancement of APMg skin penetration. Our data also suggest that iontophoretic movement of APMg predominantly occurred through the transfollicular pathway. Increased APMg penetration was also closely correlated with the duration of iontophoresis (Figure 3(b)). Taken together, these results demonstrated that iontophoresis efficiently induced APMg delivery via a transfollicular route rather than a transdermal pathway. This is due to the fact that channels in the hair follicles have a lower resistance to electric currents compared to the compact epidermal layer.

\subsection{In Vivo Iontophoretic Permeation of NaF into Hairless and Hairy Mouse Skin}

Topical delivery of $\mathrm{NaF}$ in the presence or absence of iontophoresis was performed on hairy and hairless mouse dorsal skin after electric shaving; the results are shown in Figure 4. Strong green fluorescence corresponding to $\mathrm{NaF}$ was observed by cathodic conduct through the trans- 
follicular pathway in hairy mouse skin at the telogen (7-wk-old hairy mice) and anagen (5-wk-old hairy mice) stage while the topical application only without iontophoresis showed little NaF under the skin or in hair follicles. During the anagen stage, intense NaF fluorescence was observed even in the lower fat tissues through which the relatively long transfollicular channels of anagen follicles penetrated. On the other hand, significantly less $\mathrm{NaF}$ was observed in through the epidermal layer of the hairless mouse skin. The entangled type of delivery at a shallow depth under the epidermal layer was identified.

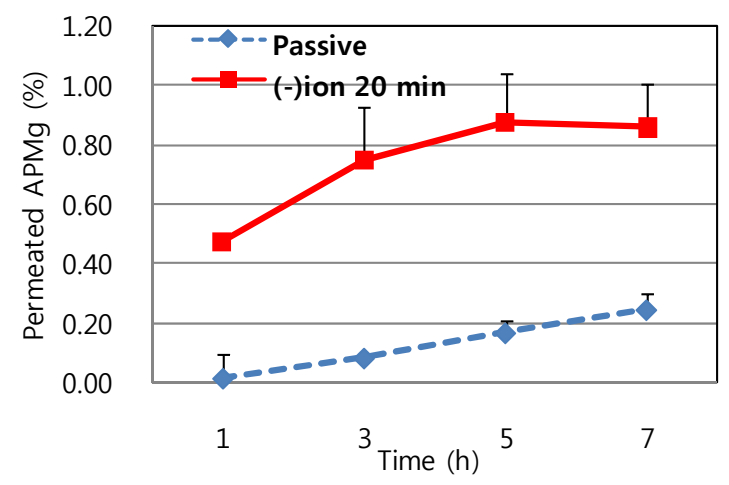

(a)

\section{Conclusions}

There are three potential routes through which a drug penetrates the scalp: follicular, non-follicular, and intercellular. Among these, the shunt follicular pathway is significantly utilized during iontophoresis. Although there are some differences among different drug types, the transfollicular pathway is the predominant route of delivery via iontophoresis rather than the transdermal pathway since the transfollicular route is less resistant to electric currents [9-12].

In the present study, the effect of iontophoresis on skin

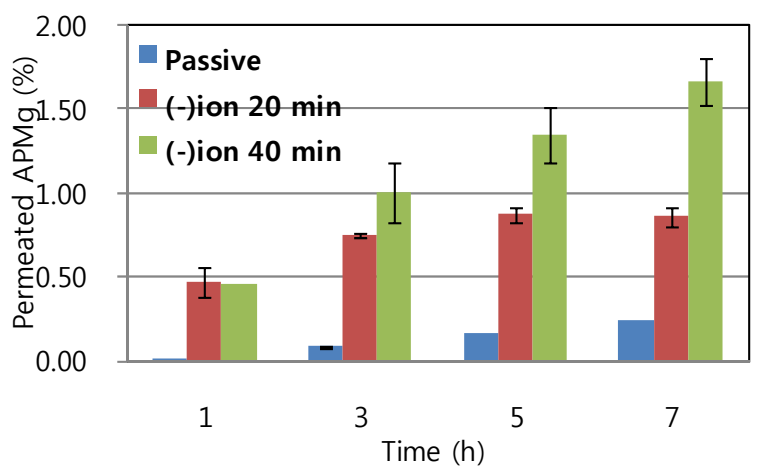

(b)

Figure 3. In vitro penetration of $0.2 \mathrm{M}$ APMg using a Franz cell equipped with hairy (telogen) skin with or without iontophoresis. A. Permeated APMg with or without iontophoresis at $-0.4 \mathrm{mV} / \mathrm{cm}^{2}$ for $20 \mathrm{~min}$, B. Effect of iontophoretic duration $(20$ and $40 \mathrm{~min}$ ). (a) Passive vs. lomtophoretic penetration; (b) Duration of iontophoresis.

Control

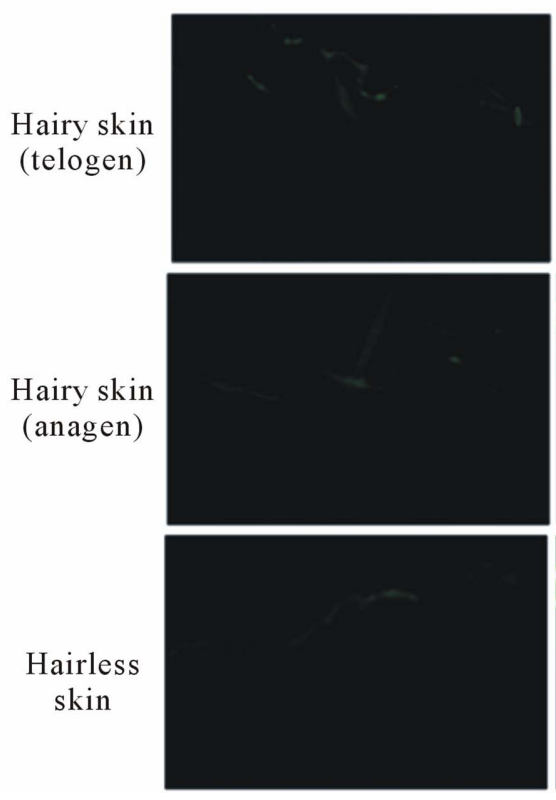

TA $1 \mathrm{~h}$
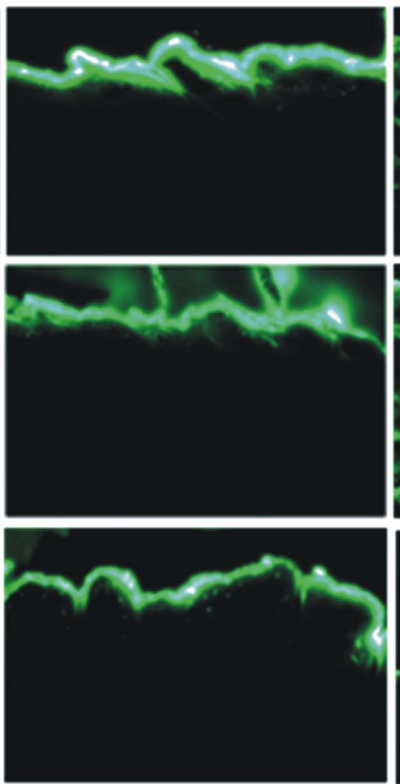

lon $20^{\prime}+$ TA $40^{\prime}$

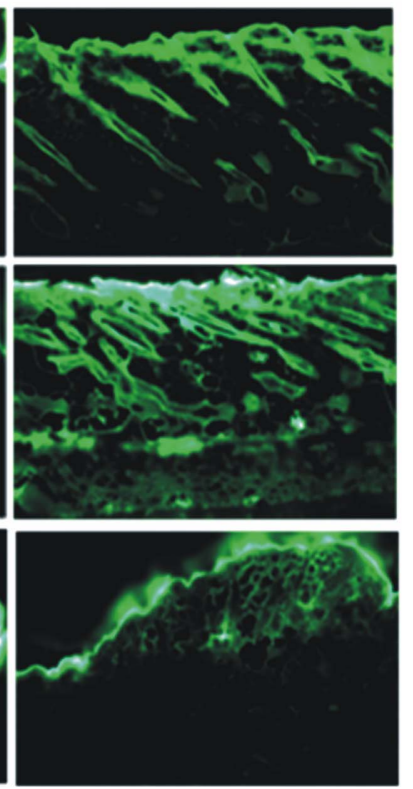

Figure 4. Penetration of $0.1 \% \mathrm{NaF}$ topically applied with or without iontophoresis on hairy or hairless mouse skin. All the magnification of this figure is 100X. Control, no treatment; TA $1 \mathrm{~h}$, topical application only for $1 \mathrm{~h}$; Ion 20' + TA 40', iontophoresis at $-0.4 \mathrm{mV} / \mathrm{cm}^{2}$ for $20 \mathrm{~min}$ and post-incubation for $40 \mathrm{~min}$; Hairy skin, shaved dorsal skin of C57BL/6 mice at the telogen (7-week-old) and anagen (5-week-old) stages; Hairless skin, dorsal skin of hairless SKH-1 mice. 
penetration of APMg was compared between hairy and hairless mouse skin. The results showed that electrotreatment is an effective method for increasing the delivery of APMg and $\mathrm{NaF}$ into hair follicles. Although iontophoresis delivery of topically applied APMg was shown to improve follicular penetration, the use of iontophoresis in the scalp requires further study since hairs are nonconductors. Nevertheless, various types of electrotreatment for skin and scalp have been evaluated not only as drug delivery modalities but also as electrotherapies [13].

\section{REFERENCES}

[1] R. Paus, S. Müller-Röver, C. Van Der Veen, et al., "A Comprehensive Guide for the Recognition and Classification of Distinct Stages of Hair Follicle Morphogenesis," Journal of Investigative Dermatology, Vol. 113, No. 4, 1999 , pp. 523-532. doi:10.1046/j.1523-1747.1999.00740.x

[2] S. Takamizawa, Y. Maehata, K. Imai, et al., "Effects of Ascorbic Acid and Ascorbic Acid 2-Phosphate, a LongActing Vitamin C Derivative, on the Proliferation and Differentiate on of Human Osteoblast-Like Cells," Cell Biology International, Vol. 28, No. 4, 2004, pp. 255-265. doi:10.1016/j.cellbi.2004.01.010

[3] M. H. Kwack, S. H. Shin, S. R. Kim, et al., "L-Ascorbic Acid 2-Phosphate Promotes Elongation of Hair Shafts via the Secretion of Insulin-Like Growth Factor-1 from Dermal Papilla Cells through Phosphatidylinositol 3-Kinase. British Journal of Dermatology, Vol. 160, No. 6, 2009, pp. 1157-1162. doi:10.1111/j.1365-2133.2009.09108.X

[4] Y. K. Sung, S. Y. Hwang, S. Y. Cha, et al., "The Hair Growth Promoting Effect of Ascorbic Acid 2-Phosphate, a Long-Acting Vitamin C Derivative," Journal of Dermatological Science, Vol. 41, No. 2, 2006, pp. 150-152. doi:10.1016/j.jdermsci.2005.11.010

[5] Y. K. Sung, M. H. Kwack, S. R. Kim, et al., "Transcriptional Activation of $\mathrm{CCN} 1$ and $\mathrm{CCN} 2$, Targets of Canonical Wnt Signal, by Ascorbic Acid 2-Phosphate in Human Dermal Papilla Cells," Journal of Dermatological Science, Vol. 49, No. 3, 2009, pp. 256-259. doi:10.1016/j.jdermsci.2007.09.005
[6] F. Marra, J. Levy, P. Santi and Y. Kalia, "In Vitro Evaluation of the Effect of Electrotreatment on Skin Permeability," Journal of Cosmetic Dermatology, Vol. 7, No. 2, 2008, pp. 105-111. doi:10.1111/j.1473-2165.2008.00372.x

[7] J. Yoo, S. Shanmugam, C. Song, et al., "Skin Penetration and Retention of L-Ascorbic Acid 2-Phosphate Using Mutilamellar Vesicles," Archives of Pharmacal Research, Vol. 31, No. 12, 2008, pp. 1652-1658. doi:10.1007/s12272-001-2164-4

[8] M. Ebihara, M. Akiyama, Y. Ohnishi, et al., "Iontophoresis Promotes Percutaneous Absorption of L-Ascorbic Acid in Rat Skin," Journal of Dermatological Science, Vol. 32, No. 3, 2003, pp. 217-222. doi:10.1016/S0923-1811(03)00105-1

[9] I. Han, M. Kim and J. Kim, "Enhanced Transfollicular Delivery of Adriamycin with a Liposome and Iontophoresis," Experimental Dermatology, Vol. 13, No. 2, 2004, pp. 86-92. doi:10.1111/j.0906-6705.2004.00123.x

[10] K. Kajimoto, M. Yamamoto, M. Watanabe, et al., "Noninvasive and Persistent Transfollicular Delivery System Using a Combination of Liposomes and Iontophoresis," International Journal of Pharmaceutics, Vol. 403, No. 1-2, 2011, pp. 57-65. doi:10.1016/j.ijpharm.2010.10.021

[11] E. R. Scott, A. I. Laplaza, H. S. White and J. B. Phipps, "Transport of Ionic Species in Skin: Contribution of Pores to the Overall Skin Conductance," Pharmaceutical Research, Vol. 10, No. 12, 1993, pp. 1699-1709. doi:10.1023/A:1018909811672

[12] B. D. Bath, E. R. Scott, P. Bradley and H. S. White, "Scanning Electrochemical Microscopy of Iontophoretic Transport in Hairless Mouse Skin. Analysis of the relative Contributions of Diffusion, Migration, and Electroosmosis to Transport in Hair Follicles," Journal of Dermatological Science, Vol. 89, No. 12, 2000, pp. 1537-1549. doi:10.1002/1520-6017(200012)89:12<1537::AID-JPS4> 3.0.CO;2-J

[13] P. Minhas, V. Bansal, J. Patel, et al., "Electrodes for High-Definition Transcutaneous DC Stimulation for Applications in Drug Delivery and Electrotherapy, including tDCS," Journal of Neuroscience Methods, Vol. 190, No. 2, 2000, pp. 188-197. doi:10.1016/j.jneumeth.2010.05.007 